\title{
Microstructure Evolution in Thin Zirconia Films: Experimental Observation and Modelling
}

\author{
X. Wang ${ }^{*}$ and A. Atkinson \\ Department of Materials, Imperial College, London SW7 2AZ, UK
}

\begin{abstract}
The gas-tightness and high ionic conductivity of the electrolyte are essential requirements for realisation of high efficiency and stable operation of solid oxide fuel cells (SOFCs). In this study, the microstructure of YSZ electrolyte films sintered on a rigid substrate was characterised using scanning electron microscopy. The pore size, shape and orientation were quantitatively determined based on image analysis of SEM micrographs. It was found that the pores are elongated and oriented preferentially perpendicular to the film plane. The average pore size was found to increase, while the pore elongation and preferential orientation decrease with sintering. A variational principle based sintering model capable of predicting microstructural evolution is presented. The modelling results agree with the experimental observations in that constrained sintering leads to an anisotropic microstructure which becomes more isotropic with densification. Decreasing grain boundary diffusion with respect to surface diffusion is predicted to produce a more isotropic pore geometry. The model also predicts that in constrained sintering there exists a critical pore size above which the pore grows instead of shrinks. It is critical to control defect size at an early stage of processing to be smaller than the critical pore size in order to obtain leak-free electrolyte films.
\end{abstract}

Keywords: Thin film; Sintering; Modelling; Anisotropic microstructure; Orientation

\footnotetext{
* Corresponding author, Tel: +44 2075496771, Fax:+44 2075946757 ,

E-mail address: xin.wang @imperial.ac.uk
} 


\section{Introduction}

Solid Oxide Fuel Cells (SOFCs) offer many advantages over other fuel cell types. SOFCs can be used with a variety of fuels from hydrogen to hydrocarbons with a minimum of fuel processing and may also play a role in carbon sequestration strategies [1,2]. A critical material for SOFCs is the electrolyte used to transport ions between the two electrodes. For example, high-performance SOFCs require a thin and gastight electrolyte membrane that is coated on a porous electrode which, in turn, could be supported on a porous substrate [3]. During fabrication, the sintering of the electrolyte films is under a constraint from the support and in some concepts, e.g. the integrated planar or segmented in series design of Rolls-Royce Fuel Cell Systems [4], the support does not shrink significantly during sintering of the electrolyte. The constraint generates a tensile stress during sintering that can lead to crack-like defects [5], delaminations and unfavourable pore orientation [6-8] in the films. If the defects and pores are large enough or penetrate the electrolyte film they can lead to lowered fuel efficiency and creation of local "hot-spots" that cause mechanical damage to the cells. However, the formation and evolution of such defects during sintering is not well understood.

Potential defects can be inherent in the green microstructure (e.g., formed during screen printing), or may be induced during sintering. Bordia and Jagota [5] postulated that cracklike defect formation in $\mathrm{Al}_{2} \mathrm{O}_{3}$ films originates from local regions of lower than average green density. Due to the anisotropic microstructure, and consequently anisotropic properties, developed in the films, isotropic continuum constitutive laws are no longer able to describe the sintering process completely [9]. On the other hand the discrete element method (DEM) has proved useful for studying crack initiation [10,11] and the structure of initial particle packing [12]. For example, Henrich et al. [10] found that particle rearrangement plays a significant role in the development of a crack in the initial stages of sintering. If rearrangement is suppressed, cracks tend to form more easily because of increased local stresses. In a recent work, Wonisch et al. [7] and Martin et al. [11] demonstrated that DEM modeling can be used to simulate anisotropic microstructure development and defect evolution in films. It was concluded that although geometrical constraint is necessary for a defect to grow into a crack, the presence of an initial defect is not a necessary condition to initiate cracks [11]. However, since DEM simulation can only deal with defect formation in the early stages of sintering, to our knowledge currently no sintering model exists which is 
capable of predicting defect evolution in the intermediate and later stages. Therefore, a sintering model which can predict defect evolution in the later stages of sintering is highly desirable. Such a model would benefit not only the understanding of constrained sintering in general, but also improve our understanding of the functional and structural properties of the electrolyte film in SOFCs and similar devices.

This contribution is separated into three parts: firstly experimental observation and quantitative information of the microstructures of thin electrolyte films are presented; secondly a sintering model based on the variational principle is developed; finally modelling results are presented and compared to the experimental results. Discussion is given with regard to the sintering mechanism, the evolution of anisotropic pore geometry and the key properties that affect the formation of the anisotropic microstructure.

\section{Experimental Details and Quantitative Image Analysis}

Yttria-stabilised zirconias (YSZ) are popular materials used for the electrolyte in SOFCs. Currently both $3 \mathrm{YSZ}$ (partially stabilised zirconia with $3 \mathrm{~mol} \% \mathrm{Y}_{2} \mathrm{O}_{3}$ ) and 8YSZ (nominally fully stabilised zirconia with $\left.8 \mathrm{~mol} \% \quad \mathrm{Y}_{2} \mathrm{O}_{3}\right)$ are used in a variety of SOFC designs. Although 8YSZ has a higher ionic conductivity than 3YSZ, 3YSZ has better mechanical properties. Therefore, both 8YSZ and 3YSZ films were studied in this work.

The film layer and substrate were chosen to be the same composition to ensure that no stresses would be generated by thermal expansion differences between the layer and substrate. The substrates were commercially produced by Kerafol GmbH (Eschenbach, Germany). The YSZ powders used to prepare the screen-printing inks for the films were supplied by MEL Chemicals, UK. The particle size distributions of the powders were measured by light scattering and the data are summarised in Table 1.

YSZ layers were deposited on the substrates (in the as-received condition) by screen printing (165 mesh screen and $2.5 \mathrm{~mm}$ gap) and the layers were oven dried at $120{ }^{\circ} \mathrm{C}$. The dried films were held at the sintering temperature for various lengths of time using a heating rate of $3^{\circ} \mathrm{C} / \mathrm{min}$ and cooled to room temperature using a cooling rate of $5^{\circ} \mathrm{C} / \mathrm{min}$. The green YSZ films were $\sim 14 \mu \mathrm{m}$ in thickness, and the sintered films were $7 \sim 9 \mu \mathrm{m}$ in thickness, depending on sintering temperature and time. 
Microstructure observation of the films was performed on thermally etched polished cross sections and untreated top surfaces with a high resolution field emission gun scanning electron microscope (Gemini LEO 1525). The images were analyzed quantitatively using the software ImageJ. In the preparation of cross-sections, the sintered samples were sectioned perpendicular to the film plane, mounted, and vacuum-impregnated with a low-viscosity epoxy resin. Resin impregnation was used to prevent pullout during subsequent grinding and polishing down to a $0.5 \mu \mathrm{m}$ diamond finish. After polishing, the sintered samples were removed from their mounts, and thermally etched at $1150{ }^{\circ} \mathrm{C}$ for 15 minutes. The thermal etching temperature and time were carefully selected to reveal the grain boundaries.

The image analysis involved first finding edges and adjusting the threshold intensity to produce a binary image. Then a combination of dilating, filling holes, eroding, close and open functions was used in order to make the processed image a best match to the original image. The pores, which are isolated in the 2-D sections, appear as dark "particles" in the processed image. They were analysed using the function 'analyse particles' of ImageJ by representing them as ellipses of equivalent area. The major and minor axes of each ellipse, and the orientation of the major axis, were determined by mathematically best fitting the pore boundary to an elliptical equation. At least 600 pores for each sample were taken into account for quantitative analysis.

Based on the image analysis, the volume fraction of porosity was calculated as the area fraction of the pores on the 2-D SEM images. The average pore elongation factor, $\varepsilon$, was calculated as the arithmetic mean of the aspect ratio of the pores $\left(\varepsilon_{\mathrm{i}}=d_{i}^{\text {maj }} / d_{i}^{\min }\right.$, with $d_{i}^{\text {maj }}$ and $d_{i}^{\min }$ being the major and minor axes of the ellipses) weighted by pore volume:

$$
\varepsilon=\frac{\sum_{i} \varepsilon_{i} d_{i}^{\text {min }}\left(d_{i}^{\text {maj }}+d_{i}^{\text {min }}\right) d_{i}^{\text {maj }}}{\sum_{i} d_{i}^{\text {min }}\left(d_{i}^{\text {maj }}+d_{i}^{\text {min }}\right) d_{i}^{m a j}} \quad \text { Eq. } 1
$$

where each pore was assumed to be an ellipsoid with three axes: $d_{i}^{\text {maj }}, d_{i}^{\min }$ and $\left(d_{i}^{m a j}+d_{i}^{\text {min }}\right) / 2$. For 2-D images, the surface can cut ellipsoids at any position instead always at the symmetrical plane. On average, the actual 3-D pore size is larger than the 2-D (observed) pore size. A geometrical analysis based on assuming randomly distributed 
ellipsoids gives a conversion factor of $4 / \pi$. Therefore, the average pore size was calculated by the arithmetic mean of $\left(d_{i}^{\text {maj }}+d_{i}^{\text {min }}\right) / 2$ weighted by pore volume and multiplied by the conversion factor:

$d_{m}=\frac{4}{\pi} \frac{\sum_{i} d_{i}^{\text {min }}\left(d_{i}^{\text {maj }}+d_{i}^{\text {min }}\right)^{2} d_{i}^{\text {maj }} / 2}{\sum_{i} d_{i}^{\text {min }}\left(d_{i}^{\text {maj }}+d_{i}^{\text {min }}\right) d_{i}^{\text {maj }}} \quad$ Eq. 2

The pore orientation was quantified using the angle $\theta_{i}$ measured between the film plane and the major axis of the ellipse. In an earlier study by Guillon et al. [6], pores with $\theta_{i}$ from $0^{\circ}$ to $30^{\circ}$ and from $150^{\circ}$ to $180^{\circ}$ were classified as "horizontal", and pores with $\theta_{i}$ from $60^{\circ}$ to $120^{\circ}$ as "vertical" [6,8]. In the present work a parameter, $k$, proposed by Mucke et al. [3] was used to characterise the pore orientation for the distribution:

$k=\ln \left(\frac{\sum_{i}\left(d_{i} \varepsilon_{i} \sin \theta_{i}\right)^{2}}{\sum_{i}\left(d_{i} \varepsilon_{i} \cos \theta_{i}\right)^{2}}\right.$

Eq. 3

In calculating $k$, all pores are included and their individual orientation is taken into account. For the sake of uniform scaling, the logarithm is used, which introduces a mathematical symmetry between vertically and horizontally aligned pores [3]. If $k=0$, there is no preferential orientation of the pores. When $k>0$, the pores are preferentially orientated perpendicular to the film plane, and when $k<0$ the pores are preferentially orientated parallel to the film plane.

Grain size was measured on thermally etched polished samples using the linear intercept method. More than five SEM images per sample were processed by the linear intercept method and a correction factor of 1.56 was used to obtain the 3-D grain size [3].

\section{Experimental Results}

Typical microstructures of the films are illustrated in Figs.1a-c. Figs.1a and b show top surface SEM images for 3YSZ and 8YSZ, respectively, which were sintered at $1350^{\circ} \mathrm{C}$ for 1h. Both 3 YSZ and 8YSZ film surfaces were quite porous at this stage. Although the pore size in the two different films was similar, the grain size in 8YSZ was much larger than that 
of 3YSZ. Fig.1c is a typical cross-section SEM image of 8YSZ which shows a microstructure similar to that seen from the top surface.

Although the grain size and porosity of the films varied depending on the sintering condition, almost all the pores in both 3YSZ and 8YSZ films were submicron in size $(<1 \mu \mathrm{m})$. Large pores (normally $5-20 \mu \mathrm{m}$ in length) did exist, but the number density of these large defects was only $\sim 100 / \mathrm{mm}^{2}$. Compared to the number density of the submicron pores $\left(\sim 10^{6} / \mathrm{mm}^{2}\right)$, the large pores were very rare. Fig. 2 shows a large defect present on the top surface of a film. A common feature of these large defects is that they were at least ten times larger than the average pore size. Defects or pores in the size range $2-5 \mu \mathrm{m}$ were not seen in either $3 \mathrm{YSZ}$ or 8YSZ films. Another observation is that the areas next to these large defects (as marked by drawn white lines in Figure 2) were better sintered (lower volume fraction of pores) than the majority of the film. Since these large defects were quite rare they were normally not present in the high magnification SEM images which were analysed using ImageJ. Therefore, images containing such defects were not included in the quantification of microstructural parameters such as porosity, pore size, pore orientation, etc.

Fig.3 shows the densification of the films with increasing sintering temperature and/or increasing time at $1350^{\circ} \mathrm{C}$. It can be seen that the density achieved for 3 YSZ was always higher than that for 8YSZ under the same sintering conditions. This means that the densification rate of 3 YSZ films is faster than that of 8YSZ. It is particularly interesting to note that densification continues for long times at the highest temperature studied and there is no indication of a limiting density that is lower than $100 \%$. The volume fractions of porosity in the top half of the film (near the free surface) and the bottom half (near the interface) were measured independently and found to be the same to within experimental error. Thus the effect of substrate constraint was uniform through the film thickness.

Fig.4 shows the evolution of the mean volume-weighted pore size as a function of relative density. For both materials, the average pore size increased with the density, but the average pore size in the 3YSZ films was slightly larger than that in 8YSZ films.

Fig. 5 shows the evolution of the average pore elongation observed from both the top surface and cross section. In both materials, the pore elongation decreased with the relative density, but the 3YSZ films had more elongated pores than the 8YSZ films. 
From Fig.5, pore elongation on the top surfaces was found to be similar to that on cross sections. From top surface images, the orientation index $k$ was typically $0 \pm 0.2$ for both 3 YSZ and 8YSZ films. Therefore, the pores, although elongated had no preferential orientation within the film plane as expected. In contrast, from cross sections, $k$ is larger than 0 for films of both materials (Fig. 6). Therefore, the pores were preferentially orientated perpendicular to the film plane in both materials. As shown in Fig.6, 3YSZ has a much larger degree of preferred orientation than 8YSZ.

The grain growth data are shown in Fig.7. 8YSZ films had much larger grain size than 3 YSZ films for a given relative density. The 8 YSZ films also exhibited an increased grain growth after the film density reached $95 \%$.

\section{Constrained sintering model based on the variational principle}

The microstructural evolution during sintering is determined by both thermodynamics and kinetics acting together. According to the variational principle, the system evolves so as to minimize a function $\Pi$, which is the sum of the rate of free energy change, $\dot{G}$, and the energy dissipation rate, $\Psi$ [13-17]. The variational principle approach has been used for studies of grain boundary cavitation by creep [13], grain boundary and surface diffusion [15], grain boundary migration $[14,16]$ and sintering of thermal barrier coatings [17, 18].

In classic sintering models [19-21], each grain has its attendant porosity, which thus requires the pore size to continually decrease and be smaller than the grain size, in the absence of grain growth. The pores are located in grain boundary triple-junctions and the diffusion length scale is the grain size $[19,21]$. The triple-junction type of pore structure obviously does not fit the real microstructure of the electrolyte films as shown in Fig.1. For example for 3 YSZ (Fig.1a), most of the pores are larger than the grains. Pore separation is larger than the grain size and one pore can be surrounded by a larger number of grains, instead of only three (as in Coble's model [19]).

In our model, the pore size has no a priori relationship to pore separation and grain size, and the pore is allowed to be surrounded by many grains. As shown in Fig. 8, this is a three dimensional model, with a representative unit containing a number of cubic grains of the same size (D) and a closed pore. During sintering, both the film thickness $(h)$ and the volume of the pore decrease with the time. The pore can be elongated vertically (prolate spheroid, with $e=b / a>1$ ) or squashed vertically (oblate spheroid with $e<1$ ). For convenience, 
'vertical' means the direction perpendicular to the film plane. However, the "horizontal" dimensions of the unit cell $(c)$ remain constant because of the in-plane constraint.

In this model, $a, e$ and $D$ are allowed to change with time independently (i.e., $\dot{a}, \dot{e}$ and $\dot{D}$ are independent variables). In contrast, the change of the thickness $h$ is dependent on the changes of $a$ and $e$, so $\dot{h}$ is not an independent variable.

Although diffusion coefficients have been determined for several different zirconias, with varying values of the pre-exponential constant $\left(D_{0}\right)$ and activation energy $(Q)$, it has been found the absolute values of the diffusion coefficients are quite similar [22] . From Fig.1 in [22] it can be seen that both the grain boundary diffusion coefficient and surface diffusion coefficient are not sensitive to yttrium content. The grain boundary diffusion coefficient is about 6 orders of magnitude higher than the lattice diffusion coefficient at sintering temperatures $[22,23]$. The ratio of grain boundary diffusion flux to lattice diffusion flux can be expressed:

$R_{d i f f}=\frac{D_{G B} \delta_{G B}}{D_{L} D} \quad$ Eq. 4

where $D_{G B}=1 \times 10^{-3} \exp (-370000 / \mathrm{RT})$ [23], $D_{L}=5 \times 10^{-4} \exp (-515000 / \mathrm{RT})$ [23] and $\delta_{G B}=0.46 \mathrm{~nm}$ [17]. $R_{\text {diff }}$ is inversely proportional to the grain size, which means lattice diffusion can become significant only when grain size becomes large. For 3YSZ grain growth is slow and even in the later stage of the sintering $D$ is smaller than $0.5 \mu \mathrm{m}$. In this case, $R_{\text {diff }}$ is estimated to be larger than 100 (at sintering temperatures $1100-1350{ }^{\circ} \mathrm{C}$ ). For $8 \mathrm{YSZ}$, although $D$ can become as large as $5 \mu \mathrm{m}$ in the later stage of sintering, $R_{\text {diff }}$ can still be larger than 10 . Therefore, in both YSZ compositions, the grain boundary should be the rate controlling transport pathway for sintering.

Due to the constraint of the substrate, there is no horizontal shrinkage (in-plane shrinkage). Hence we assume that diffusion along all the vertical grain boundaries is fully suppressed and all the matter transported into the pore is via horizontal grain boundaries. (In reality boundaries will be inclined, but can be considered as having vertical and horizontal components.)

The variational principle requires $\Pi=\dot{G}+\Psi$ to be minimum with respect to the independent 
variables. Therefore the governing equations for evolution of the system are:

$\frac{\partial \dot{G}}{\partial \dot{D}}+\frac{\partial \Psi}{\partial \dot{D}}=0 \quad$ Eq. 5

$\frac{\partial \dot{G}}{\partial \dot{a}}+\frac{\partial \Psi}{\partial \dot{a}}=0 \quad$ Eq. 6

$\frac{\partial \dot{G}}{\partial \dot{e}}+\frac{\partial \Psi}{\partial \dot{e}}=0 \quad$ Eq. 7

The free energy $G$ is a sum of the stored elastic strain energy, the total grain boundary energy and the total surface energy:

$$
G=U^{E}+\int_{A s} \gamma_{S} d A_{s}+\int_{A_{G B}} \gamma_{G B} d A_{G B} \quad \text { Eq. } 8
$$

where $\gamma_{\mathrm{s}}$ and $\gamma_{\mathrm{gb}}$ are the surface energy and grain boundary energy. Hence the rate of free energy change is a function of the time derivatives of pore size $(\dot{a})$, pore shape $(\dot{e})$ and grain size $(\dot{D})$. In what follows we give the results obtained for these functions for the geometry used in the model. Detailed derivations can be found in the supporting information. For the case of a prolate spheroid:

$$
\begin{aligned}
& \dot{G}=\gamma_{s}\left[4 \pi a \dot{a}+\frac{4 \pi a e^{2} \dot{a}}{\sqrt{e^{2}-1}} \arcsin \left(\frac{\sqrt{e^{2}-1}}{e}\right)+2 \pi a^{2}\left(\frac{2 e}{\sqrt{e^{2}-1}}-\frac{e^{3}}{\sqrt{\left(e^{2}-1\right)^{3}}}\right) \arcsin \left(\frac{\sqrt{e^{2}-1}}{e}\right) \dot{e}+\frac{2 \pi a^{2} e \dot{e}}{e^{2}-1}\right] \\
& +\gamma_{g b}\left[\frac{2 c^{2} \dot{h}}{D}+\frac{4 \pi D \dot{D}}{e^{2}} \sum_{i=1}^{N_{H}} i^{2}+\left(4 \pi D^{2} \dot{e}+8 \pi e D \dot{D}\right) \sum_{i=1}^{N_{V}} i^{2}-2 \pi a \dot{a}\left(2 N_{H}+1\right)-4 \pi e a \dot{a}\left(2 N_{V}+1\right)-\right. \\
& \left.2 \pi a^{2} \dot{e}\left(2 N_{V}+1\right)-\frac{4 \pi D^{2} \dot{e}}{e^{3}} \sum_{i=1}^{N_{H}} i^{2}-\frac{2 c^{2} h \dot{D}}{D^{2}}-\frac{c^{3} \dot{D}}{D^{2}}\right]+\dot{U}^{E}
\end{aligned}
$$

Eq. 9

And for the oblate spheroid case:

$$
\begin{aligned}
& \dot{G}=\gamma_{s}\left[4 \pi a \dot{a}+\frac{4 \pi a e^{2} \dot{a}}{\sqrt{1-e^{2}}} \operatorname{Ln}\left(\frac{1+\sqrt{1-e^{2}}}{e}\right)+2 \pi a^{2}\left(\frac{2 e}{\sqrt{1-e^{2}}}+\frac{e^{3}}{\sqrt{\left(1-e^{2}\right)^{3}}}\right) \operatorname{Ln}\left(\frac{1+\sqrt{1-e^{2}}}{e}\right) \dot{e}-\frac{2 \pi a^{2} e \dot{e}}{1-e^{2}}\right] \\
& +\gamma_{g b}\left[\frac{2 c^{2} \dot{h}}{D}+\frac{4 \pi D \dot{D}}{e^{2}} \sum_{i=1}^{N_{H}} i^{2}+\left(4 \pi D^{2} \dot{e}+8 \pi e D \dot{D}\right) \sum_{i=1}^{N_{V}} i^{2}-2 \pi a \dot{a}\left(2 N_{H}+1\right)-4 \pi e a \dot{a}\left(2 N_{V}+1\right)-\right. \\
& \left.2 \pi a^{2} \dot{e}\left(2 N_{V}+1\right)-\frac{4 \pi D^{2} \dot{e}}{e^{3}} \sum_{i=1}^{N_{H}} i^{2}-\frac{2 c^{2} h \dot{D}}{D^{2}}-\frac{c^{3} \dot{D}}{D^{2}}\right]+\dot{U}^{E}
\end{aligned}
$$


Eq. 10

where $2 N_{V}+1$ is the total number of vertical grain boundaries and $2 N_{H^{+}} 1$ is the total number of horizontal grain boundaries intersecting the pore. Based on a finite element analysis (the details are not presented here to save space) the stored strain energy is about 3 orders of magnitude smaller than the surface energy and grain boundary energy in the system, and therefore $\dot{U}^{E}$ can be neglected.

The processes that can dissipate energy during sintering are 1) grain boundary diffusion; 2) surface diffusion along the inner surface of the pore and 3) grain boundary migration [17].

$\Psi=\int_{A_{S}} \frac{j_{S}^{2}}{2 M_{S} \Omega \delta_{S}} d A_{S}+\int_{A_{G B}} \frac{j_{G B}^{2}}{2 M_{G B} \Omega \delta_{G B}} d A_{G B}+\int_{A_{G B}} \frac{v_{m}^{2}}{2 m_{m}} d A_{G B} \quad$ Eq. 11

where $j$ is the volume flux in unit length of diffusion front along free surface $\left(j_{S}\right)$ or grain boundary $\left(j_{G B}\right) . v_{m}$ is the grain boundary migration velocity, $A_{S}$ and $A_{G B}$ are surface area and grain boundary area, respectively. $M$ is the atomic mobility [24]:

$$
M=\frac{D_{o} \exp (-Q / R T)}{k_{B} T} \quad \text { Eq. } 12
$$

$m_{m}$ is the intrinsic grain boundary mobility [24]:

$m_{m}=\frac{D_{G B o} \exp \left(-Q_{G B} / R T\right)}{k_{B} T} \frac{\Omega}{\delta_{G B}} \quad$ Eq. 13

and $\Omega$ is the molecular volume. For the microstructural model shown in Fig.8, the total energy dissipation rate by grain boundary diffusion can be written as:

$\Psi_{G B}=\frac{\dot{h}_{o} F_{o}}{16 M_{G B} \Omega \delta_{G B}}+2 \sum_{i=i}^{N_{H}} \frac{\dot{h}_{i} F_{i}}{16 M_{G B} \Omega \delta_{G B}} \quad$ Eq. 14

where $F_{i}=c^{4}\left[\frac{4}{3} \operatorname{Ln}\left(\frac{c}{\sqrt{a^{2}-i^{2} D^{2} e^{-2}}}\right)+\frac{\pi}{6}-\frac{16}{9}-\frac{2 \operatorname{Ln} \sqrt{2}}{3}\right]+4 c^{2}\left(a^{2}-i^{2} D^{2} e^{-2}\right)-\pi\left(a^{2}-i^{2} D^{2} e^{-2}\right)$

The energy dissipation rate by surface diffusion along the inner surface of the pore is: 


$$
\begin{aligned}
& \Psi_{S}=\sum_{i=1}^{N_{V}}\left[\frac{1}{2 \pi M_{S} \Omega \delta_{S}} \int_{(i-1) D}^{i D} \frac{\left(j_{S i}^{V}(z)\right)^{2} \sqrt{a^{2}+z^{2}\left(e^{-4}-e^{-2}\right)}}{\left(a^{2}-z^{2} e^{-2}\right)} d z\right]+ \\
& \frac{1}{2 \pi M_{S} \Omega \delta_{S}} \int_{i D}^{a e} \frac{\left(j_{S i}^{V}(z)\right)^{2} \sqrt{a^{2}+z^{2}\left(e^{-4}-e^{-2}\right)}}{\left(a^{2}-z^{2} e^{-2}\right)} d z
\end{aligned}
$$

where

$$
j_{S i}^{V}(z)=\left(c^{2}-\pi a^{2}+\frac{\pi z^{2}}{e^{2}}\right) \sum_{n=1}^{i}\left(\frac{\dot{h}_{o}}{2}+\dot{h}_{n}\right)-2 \pi a z \dot{a}-\frac{2 \pi z^{3}}{3 e^{3}} \dot{e} \quad \text { Eq. } 16
$$

The energy dissipation rate by grain boundary migration is :

$\Psi_{m i g}=\frac{\dot{D}^{2} A_{G B}}{8 m_{m}} \quad$ Eq. 17

where

$$
A_{G B}=\left[\frac{c^{3}}{D}+\frac{2 c^{2} h}{D}+\frac{2 \pi D^{2}}{e^{2}} \sum_{i=1}^{N_{H}} i^{2}+4 \pi e D^{2} \sum_{i=1}^{N_{V}} i^{2}-\pi a^{2}\left(2 N_{H}+1\right)-2 \pi e a^{2}\left(2 N_{V}+1\right)\right] \text { Eq. } 18
$$

From Eqs.9-10 free energy change rate can be expressed as:

$\dot{G}=\frac{\partial \dot{G}}{\partial \dot{a}} \dot{a}+\frac{\partial \dot{G}}{\partial \dot{e}} \dot{e}+\frac{\partial \dot{G}}{\partial \dot{D}} \dot{D}$

where $\frac{\partial \dot{G}}{\partial \dot{a}}, \frac{\partial \dot{G}}{\partial \dot{e}}$ and $\frac{\partial \dot{G}}{\partial \dot{D}}$ are independent of $\dot{a}, \dot{e}, \dot{D}$ (refer to Table 2).

Meanwhile from Eqs.14-18, the total energy dissipation rate can be expressed as:

$\Psi=\Psi_{G B}+\Psi_{S}+\Psi_{m i g}=\frac{\partial^{2} \Psi}{\partial \dot{a}^{2}} \dot{a}^{2}+2 \frac{\partial^{2} \Psi}{\partial \dot{a} \partial \dot{e}} \dot{a} \dot{e}+\frac{\partial^{2} \Psi}{\partial \dot{e}^{2}} \dot{e}^{2}+\frac{\partial^{2} \Psi}{\partial \dot{D}^{2}} \dot{D}^{2}$

where $\frac{\partial^{2} \Psi}{\partial \dot{a}^{2}}, \frac{\partial^{2} \Psi}{\partial \dot{e}^{2}}, \frac{\partial^{2} \Psi}{\partial \dot{e} \partial \dot{a}}$ and $\frac{\partial^{2} \Psi}{\partial \dot{D}^{2}}$ are independent of $\dot{a}, \dot{e}, \dot{D}$ (refer to Table 2).

Therefore, the microstructure changes (i.e., $\dot{a}, \dot{e}, \dot{D}$ ) can be predicted by numerically solving the following system of equations: 


$$
\left[\begin{array}{ccc}
\frac{\partial^{2} \Psi}{\partial \dot{a}^{2}} & \frac{\partial^{2} \Psi}{\partial \dot{a} \partial \dot{e}} & 0 \\
\frac{\partial^{2} \Psi}{\partial \dot{a} \partial \dot{e}} & \frac{\partial^{2} \Psi}{\partial \dot{e}^{2}} & 0 \\
0 & 0 & \frac{\partial^{2} \Psi}{\partial \dot{D}^{2}}
\end{array}\right]\left[\begin{array}{c}
\dot{a} \\
\dot{e} \\
\dot{D}
\end{array}\right]=\left[\begin{array}{c}
-\frac{\partial \dot{G}}{\partial \dot{a}} \\
-\frac{\partial \dot{G}}{\partial \dot{e}} \\
-\frac{\partial \dot{G}}{\partial \dot{D}}
\end{array}\right] \quad \text { Eq. } 19
$$

The analytical formulae for calculating all the elements in Eq.19 are given in Table 2. The material property input data required for the modelling are listed in Table 3.

\section{Discussion}

\subsection{Grain growth}

Grain growth is a thermally activated process and can be described by a grain growth master curve [25]:

$$
G_{t}^{m}-G_{o}^{m}=m \int_{0}^{t} k_{o} \exp \left(\frac{-Q_{G}}{R T}\right) d t \quad \text { Eq. } 20
$$

where $G_{t}$ is the instantaneous grain size, $G_{o}$ is the crystallite size of the starting powder at room temperature, $k_{o}$ is a diffusion-related constant, $Q_{G}$ is the activation energy of grain growth, $R$ is the gas constant, $T$ is absolute temperature and $m$ is the grain size exponent. Using Eq. 20 to fit the experimental results (Fig.7) resulted in the master curve parameters as listed in Table 4. The activation energy for 3YSZ is very similar to that for 8YSZ: 327 $\mathrm{kJ} / \mathrm{mol}$ for $3 \mathrm{YSZ}$ and $331 \mathrm{~kJ} / \mathrm{mol}$ for $8 \mathrm{YSZ}$, despite grain growth of $8 \mathrm{YSZ}$ being much faster than that for 3YSZ. The activation energy for grain growth is comparable to that for grain boundary diffusion $(370 \mathrm{~kJ} / \mathrm{mol})$, but significantly smaller than that for lattice diffusion $(515$ $\mathrm{kJ} / \mathrm{mol}$ ) [23]. Therefore, it can be concluded that for both 3YSZ and 8YSZ it is grain boundary diffusion rather than lattice diffusion that controls grain growth. 3 YSZ has a much larger grain size exponent $(m=4)$ than 8YSZ $(m=2)$. This is mainly due to solute segregation and sluggish phase partitioning in 3 YSZ which inhibit grain growth $[26,27]$.

\subsection{Pore preferential orientation}


Fig.9 gives two examples of how pores are predicted to change at $1350{ }^{\circ} \mathrm{C}$ according to the sintering model: one pore is initially an ideal spheroid and the other an oblate spheroid. The gap between the lines is 10 seconds. From Fig.9a, it can be seen that the initially spherical pore shrinks mainly in its horizontal dimension (in the film plane), and hardly at all in its vertical dimension. As a result an initially spherical pore would evolve into a vertically extended spheroid (prolate spheroid). For an originally oblate spheroidal pore (Fig.9b), the pore dimension actually increases in the vertical direction while horizontally it decreases with time. Therefore, the sintering model predicts pores preferentially elongated normal to the film plane which is consistent with our experimental observation.

According to our modelling results, the degree of elongation is very sensitive to the relative rates of grain boundary and surface diffusion. Although the actual relative diffusion rates are not known at present, the sensitivity to the relative diffusion rate was explored by changing the activation energy of surface diffusion. The activation energy $\left(Q_{s}\right)$ for surface diffusion in YSZ is very inconsistent in the literature, $314 \mathrm{~kJ} / \mathrm{mol}$ in [28], but $531 \mathrm{~kJ} / \mathrm{mol}$ in [29]. Fig.10 illustrates how the predicted pore shape evolution (of an initially spherical pore) is sensitive to the relative rates of grain boundary and surface diffusion. $\left(R_{D F}\right.$ is the ratio of $D_{G B} / D_{S}$. $)$ Fig.10 clearly shows a larger $R_{D F}$ is predicted to lead to a larger aspect ratio, $e$. This is because the constraint of the substrate inhibits transport into the pore from vertical grain boundaries. Therefore when grain boundary diffusion is relatively fast, the matter preferentially transported into the pore in the equatorial regions cannot be carried away over the pore surface quickly enough by the relatively slower surface diffusion in order to maintain its shape as spherical (which would minimise its surface energy). Thus the pore shape becomes elongated vertically to adapt to this kinetic limitation. On the other hand, if grain boundary diffusion is relatively slow $\left(R_{D F}\right.$ is small), surface diffusion is able to distribute material over the pore surface irrespective if where it enters the pore. Therefore, in this case the pore shape would tend to become more spheroidized. It is also clear that a longer diffusion path (as in the later stage of sintering) would slow down diffusion along the grain boundaries, and therefore would favour spheroidization of the pore. This is why the elongated pore in the intermediate stage as shown in Fig.10 would become more spheroidized in the later stage. Conversely a shorter diffusion path (as in an earlier stage of sintering) would favour the formation of a vertically elongated pore. These model predictions are in agreement with our experimental observation as summarised in Fig.6, 
which shows that the degree of preferential orientation of the pores was more severe in the earlier stage of sintering and decreased in the later stages for both 3YSZ and 8YSZ.

\subsection{Pore elongation}

Fig.5 shows the evolution of average pore elongation observed from the top surfaces and cross sections of the films. By comparing Fig.5 and Fig.6, one can see common features. Both pore elongation and pore orientation show a decreasing trend with sintering, and in each case $3 \mathrm{YSZ}$ is more susceptible to the formation of anisotropy. In the case of the cross sections the evolution of pore elongation is consistent with the model in that the constraint leads to pore elongation perpendicular to the external constraint and the stress it induces. The pores on top surface are also subjected to a bi-axial stress as a result of the constraint, so it is not surprising to see pore elongation in random directions (i.e. no preferred orientation) on the top surface. This is similar to the random direction of cracks that form in a thin film under biaxial tensile stress resulting from thermal expansion mismatch with a substrate or wet particulate films dried on a substrate. We speculate that if a pore is perfectly spherical and its microstructural environment perfectly isotropic then it would remain spherical. However, this perfection is never the case in reality and once the perfect symmetry is broken the local stress is no longer biaxial and the pore shape evolves to become elongated, but in a random orientation. Nevertheless, it is still an interesting finding that the observed pore elongation should be similar on both top surfaces and cross sections.

The reason for $8 \mathrm{YSZ}$ being less prone to the formation of an anisotropic pore structure might be due to the relatively slow grain boundary diffusion rate of $8 \mathrm{YSZ}$ as compared to that of $3 \mathrm{YSZ}$. It has been reported that an increase of $\mathrm{Y}_{2} \mathrm{O}_{3}$ doping leads to higher activation energy for grain boundary diffusion and hence a lower densification rate [30]. In addition solute segregation in tetragonal $3 \mathrm{YSZ}$ was found to inhibit grain growth $[22,26,31]$ and therefore accelerate matter transport along the grain boundary network [17]. Our experimental results in Fig.7 show a much smaller grain size in 3YSZ than in 8YSZ, and those in Fig.3 show 3YSZ has a faster densification rate than 8YSZ. These observations are consistent with grain boundary diffusion in 8YSZ being slower than in 3 YSZ. Assuming surface diffusion rates are similar in both materials, the relatively slower grain boundary diffusion rate $\left(R_{D F}\right)$ of 8YSZ would lead to a smaller pore elongation. Therefore, the anisotropy of microstructure of $8 \mathrm{YSZ}$ can be expected to be less severe than that of $3 \mathrm{YSZ}$ according to the 
model prediction in agreement with the experimental observations.

From a ceramic processing perspective, it is possible to minimise anisotropy in the microstructure by increasing the relative surface diffusion rate (by, for example, changing sintering atmosphere) and/or decreasing the relative grain boundary diffusion rate (by, for example, sintering at lower temperature or by addition of suitable dopants).

\subsection{Pore size evolution}

Fig.11 shows the evolution of pore volume (of initially spherical pores) predicted by the sintering model. It is not surprising to see that the smaller pores shrink much faster than the larger pores. This is because small pores have larger driving forces for sintering and also implies the small pores would be preferentially eliminated during sintering. This is consistent with the experimental results in Fig.4, which shows the average pore size increases with sintering, partly as a result of the preferential elimination of the small pores. What is more interesting in Fig.11 is that some large pores (diameter $=1.2$ ) tend to grow instead of shrink. This is in contrast to the case of free sintering where all pores are expected to shrink regardless of their size [32, 33]. A classic theory of Kingery and Francois [34] predicted that, for free sintering, there exists a critical coordination number above which a pore would grow instead of shrink. This was recently proved to be incorrect by Pan et al [32, 33] who showed that for free sintering all pores are predicted to shrink. However, from our model we conclude that for constrained sintering there does exist a critical pore size above which a pore would grow instead of shrink. According to the model this critical pore size is $d>12 D$ for YSZ, where $d$ is the diameter of the pore and $D$ the grain size.

To prove whether or not larger pores do increase in size during sintering we can observe experimentally the evolution of the larger pores. Fig.12a shows how larger defects evolve during sintering. Fig.12a gives the microstructure of a sample in an area having many larger defects after the sample was sintered at $1200^{\circ} \mathrm{C}$ for $5 \mathrm{~min}$. Fig. $12 \mathrm{~b}$ gives the microstructure of the same sample in the same area after the specimen was re-sintered at $1300 \mathrm{C}$ for $5 \mathrm{~min}$. It is clear that all the larger defects grew as a result of further sintering.

The grain size of 8 YSZ is much larger than that of $3 \mathrm{YSZ}$, which means the critical pore size of $8 Y S Z$ is larger than that of $3 \mathrm{YSZ}$. Therefore, the pores in $8 \mathrm{YSZ}$ are more likely to shrink than those in 3YSZ, because more pores in 8YSZ are smaller than the critical pore size than 
those in 3 YSZ. This explains why the average pore size of $3 \mathrm{YSZ}$ is larger than that of $8 \mathrm{YSZ}$ (as shown in Fig.4).

The critical pore size concept may also be used to explain the gap in the pore size distribution in the range $2-5 \mu \mathrm{m}$ in sintered YSZ films. The grain size is about $0.2-0.3 \mu \mathrm{m}$ in the earlier stage of the sintering and therefore the critical pore size is estimated to be 2.43.6 $\mu \mathrm{m}$. Therefore, the original defects within this range would either shrink to be smaller than $2 \mu \mathrm{m}$ or grow to be larger than $4 \mu \mathrm{m}$ thus creating a gap in the pore-size distribution. For the manufacturing of leak-free electrolyte films, which might be only $5 \mu \mathrm{m}$ thick, in fuel cells it is critical to prevent the formation of large through thickness defects. Our constrained sintering model indicates that to achieve this it is necessary to ensure that the initial defect size is smaller than the critical pore size.

\subsection{Quantitative comparison between modelling and experimental results}

The current variational principle model (as in Fig.8) is based on a closed pore microstructure. Therefore, it is directly applicable to the sintering in the later stages when the pores are mostly closed. The modelling outcome may also be applied qualitatively to the earlier stage of sintering, but for the purpose of quantitative comparison, it is appropriate to focus on the period when the pores become closed. It is generally accepted that the pores would become closed when the density gets near $85 \%$. For this reason, our quantitative modelling was focused on the pore elongation evolution during isothermal sintering at $1350{ }^{\circ} \mathrm{C}$ when density changes from $85 \%$ to $98 \%$ (corresponding dwelling time $=0 \sim 50 \mathrm{~h}$ )

According to experimental results for 3YSZ, the initial pore elongation $\varepsilon$ is 2.4 (Fig.5 at density $=85 \%$ ), and the initial grain size is $0.24 \mu \mathrm{m}$ (Fig.7 at density $=85 \%$ ). For the quantitative modelling, the initial pore size distribution is also needed. Strictly speaking the 3-D pore size distribution cannot be precisely measured from $2 \mathrm{D}$ cross sections. However, it was found that the pore elongation (defined in Eq.1) is not sensitive to the pore size distribution. Therefore the pore size distribution is assumed to be that in Fig.13a. which is based on 2 dimensional image analysis.

Except for $\mathrm{Q}_{\mathrm{s}}, \xi_{\mathrm{imp}}$ and $\xi_{\mathrm{m}}$, all the other input data required for the model are fixed as in Table 3. The activation energy for surface diffusion $\mathrm{Q}_{\mathrm{s}}$ is not fixed, partly because the surface diffusion data are sparse and inconsistent in the literature. More importantly, pore shape change is very sensitive to relative diffusion rate between grain boundary and 
surface. By changing the activation energy of surface diffusion, we can look at the influence of the relative diffusion rate on pore shape change. The correction factors for sintering rate ( $\left.\xi_{\text {imp }}\right)$ and grain growth $\left(\xi_{\mathrm{m}}\right)$ are expected to be strongly influenced by the powder chemistry and powder processing. Instead of being fixed in value, $\xi_{\text {imp }}$ and $\xi_{\mathrm{m}}$ were determined by fitting the model to the experimental results of densification kinetics as in Fig.1 and grain growth kinetics as in Fig.7, respectively.

Assuming all the pores are preferentially oriented perpendicular to the substrate, the evolution of pore elongation with density predicted by the model is given in Fig.13b. The predicted pore elongation change is very much dependent on the relative surface to grain boundary diffusion rate $\mathrm{R}_{\mathrm{DF}}$. With a small $\mathrm{R}_{\mathrm{DF}}$ the pores would quickly spheroidise, whereas with a large $\mathrm{R}_{\mathrm{DF}}$, the microstructural anisotropy would increase all the way to the last stage of sintering. The star symbols in Fig. 13b are the experimental observation of the pore elongation on cross sections of 3 YSZ films. The experimental results fit with the modelling results for $R_{D F}=1$. This indicates that the grain boundary diffusion coefficient of could be very close to that of surface diffusion for $3 \mathrm{YSZ}$ at $1350^{\circ} \mathrm{C}$.

\section{Summary and Conclusions}

Anisotropic pore microstructure was observed in both $3 \mathrm{YSZ}$ and $8 \mathrm{YSZ}$ films during constrained sintering, with the pores elongated and oriented preferentially perpendicular to the film plane. 3YSZ films had higher degrees of pore elongation and preferred orientation than 8YSZ films. Meanwhile the degree of pore elongation and orientation decreased with densification in both YSZ films.

The average pore size increased with densification in both films, but the average pore size of 3 YSZ was larger than that of 8 YSZ. Pores in both films were either smaller than $2 \mu \mathrm{m}$ or larger than $5 \mu \mathrm{m}$. No pores were within the range $2-5 \mu \mathrm{m}$ during the later stages of sintering.

A model for constrained sintering based on the variational principle was developed and predicts that pores become elongated and vertically oriented. The degree of elongation and orientation of the pores is sensitive to the relative rates of grain boundary and surface diffusion. The anisotropy is predicted to increase with the grain boundary diffusion rate and decrease with increasing surface diffusion rate. The modelling results are consistent with the experimental results. The more isotropic microstructure observed in both 3YSZ and 8YSZ films in the later stages of sintering can be attributed to the fact that the diffusion path 
becomes longer in the later sintering stage which slows the grain boundary diffusion flux. The observation that 8 YSZ films have a more isotropic microstructure than 3 YSZ may be attributed to its slower grain boundary diffusion rate relative to that of 3 YSZ.

The constrained sintering model predicts that there exists a critical pore size above which pores grow instead of shrink. The existence of this critical pore size explains why 8YSZ had a smaller average pore size than 3 YSZ and why the pore size distribution has a gap in the range $2-5 \mu \mathrm{m}$.

From viewpoint of processing, it is important to increase the surface diffusion rate relative to the grain boundary diffusion rate to reduce the anisotropy of the microstructure. It is also critical to control the initial defect size to be smaller than the critical pore size in order to ensure that pores do not grow and potentially penetrate the film thickness.

\section{Acknowledgements}

The authors are grateful to colleagues at Rolls-Royce Fuel Cell Systems for assistance with screen printing.

This research was carried out as part of the UK Supergen consortium project on "Fuel Cells: Powering a Greener Future". The Energy Programme is an RCUK cross-council initiative led by EPSRC and contributed to by ESRC, NERC, BBSRC and STFC.

\section{References}

[1] L. Blum, R. Steinberger-Wilckens, W. A. Meulenberg and H. Nabielek, in NATO Advanced Research Workshop on Fuel Cell Technologies, edited by N. Sammes, A. Smirnova and O. Vasylyev (Springer, Kiev, UKRAINE, 2004) p. 107.

[2] L. Blum, W. A. Meulenberg, H. Nabielek and R. Steinberger-Wilckens, Int. J. Appl. Ceram. Technol. 2 (2005) 482.

[3] R. Mucke, N. H. Menzler, H. P. Buchkremer and D. Stover, J. Am. Ceram. Soc. 92 (2009) S95. 
[4] P. Costamagna, A. Selimovic, M. Del Borghi and G. Agnew, Chem. Eng. J. 102 (2004) 61.

[5] R. K. Bordia and A. Jagota, J. Am. Ceram. Soc. 76 (1993) 2475.

[6] O. Guillon, L. Weiler and J. Rodel, J. Am. Ceram. Soc. 90 (2007) 1394.

[7] A. Wonisch, O. Guillon, T. Kraft, M. Moseler, H. Riedel and J. Rodel, Acta Mater. 55 (2007) 5187.

[8] J. B. Ollagnier, O. Guillon and J. Rodel, J. Am. Ceram. Soc. 90 (2007) 3846.

[9] R. K. Bordia, R. Z. Zuo, O. Guillon, S. M. Salamone and J. Rodel, Acta Mater. 54 (2006) 111.

[10] B. Henrich, A. Wonisch, T. Kraft, M. Moseler and H. Riedel, Acta Mater. 55 (2007) 753.

[11] C. Martin, H. Camacho-Montes, L. Olmos, D. Bouvard and R. K. Bordia, J. Am. Ceram. Soc. 92 (2009) 1435.

[12] A. Wonisch, T. Kraft, M. Moseler and H. Riedel, J. Am. Ceram. Soc. 92 (2009) 1428.

[13] A. Needleman and J. R. Rice, Acta Metallurgica 28 (1980) 1315.

[14] J. Pan, A. C. F. Cocks and S. Kucherenko, Proc. R. Soc. London Ser. A-Math. Phys. Eng. Sci. 453 (1997) 2161.

[15] Z. Suo and W. Wang, Journal of Applied Physics 76 (1994) 3410.

[16] A. C. F. Cocks and S. P. A. Gill, Acta Mater. 44 (1996) 4765.

[17] A. Cipitria, I. O. Golosnoy and T. W. Clyne, Acta Mater. 57 (2009) 980.

[18] A. Cipitria, I. O. Golosnoy and T. W. Clyne, Acta Mater. 57 (2009) 993.

[19] R. L. Coble, Journal of Applied Physics 32 (1961) 787.

[20] J. H. Zhao and M. P. Harmer, J. Am. Ceram. Soc. 71 (1988) 113.

[21] J. Svoboda and H. Riedel, Acta Metall. Mater. 43 (1995) 499.

[22] A. H. Chokshi, Scr. Mater. 48 (2003) 791.

[23] S. Swaroop, M. Kilo, C. Argirusis, G. Borchardt and A. H. Chokshi, Acta Mater. 53 (2005) 4975.

[24] M. N. Rahaman, Ceramic Processing and Sintering (Marcel Dekker, 2003).

[25] M. Mazaheri, A. Simchi, M. Dourandish and F. Golestani-Fard, Ceram. Int. 35 (2009) 547.

[26] T. Stoto, M. Nauer and C. Carry, J. Am. Ceram. Soc. 74 (1991) 2615.

[27] A. A. Sharif and M. L. Mecartney, Acta Mater. 51 (2003) 1633.

[28] A. Cipitria, in "PhD thesis" (University of Cambridge, 2008).

[29] Akash and M. J. Mayo, J. Mater. Sci. 35 (2000) 437.

[30] K. Matsui, K. Tanaka, T. Yamakawa, M. Uehara, N. Enomoto and J. Hojo, J. Am. Ceram. Soc. 90 (2007) 443.

[31] M. M. R. Boutz, A. J. A. Winnubst and A. J. Burggraaf, J. European Ceram. Soc. 13 (1994) 89.

[32] J. Pan, H. N. Ch'ng and A. C. F. Cocks, Mech. Mater. 37 (2005) 705.

[33] J. Z. Pan, A. Cocks, J. Rodel, R. Y. Huang and H. N. Ch'ng, J. Am. Ceram. Soc. 92 (2009) 1414.

[34] W. D. Kingery and B. Francois, in "Sintering Related Phenomena", edited by N. A. H. G. C. Kuczynski, and G. F. Gibbon (Goedon and Breach, New York, 1967) p. 471. 
Table 1 Particle size analysis of the YSZ powders.

\begin{tabular}{|l|l|l|}
\hline & $3 Y S Z$ & $8 Y S Z$ \\
\hline$d_{10}(\mu \mathrm{m})$ & 0.38 & 0.13 \\
\hline$d_{50}(\mu \mathrm{m})$ & 0.50 & 0.44 \\
\hline$d_{90}(\mu \mathrm{m})$ & 0.77 & 1.87 \\
\hline
\end{tabular}


Table 2 Analytical formulae for calculating contributions to the variational model.

\begin{tabular}{|c|c|c|}
\hline$\frac{\partial \dot{G}}{\partial \dot{a}}$ & \multicolumn{2}{|c|}{$\begin{array}{l}\text { For prolate spheroid } \\
\frac{\partial \dot{G}}{\partial \dot{a}}=\gamma_{s}\left[4 \pi a+\frac{4 \pi a e^{2}}{\sqrt{e^{2}-1}} \sin ^{-1}\left(\frac{\sqrt{e^{2}-1}}{e}\right)\right]+\gamma_{g b}\left[\frac{8 \pi a^{2} e}{D}-2 \pi a(2 N H+1)-4 \pi e a(2 N V+1)\right] \\
\text { For oblate spheroid } \\
\frac{\partial \dot{G}}{\partial \dot{a}}=\gamma_{s}\left[4 \pi a+\frac{4 \pi a e^{2}}{\sqrt{1-e^{2}}} \operatorname{Ln}\left(\frac{1+\sqrt{1-e^{2}}}{e}\right)\right]+\gamma_{g b}\left[\frac{8 \pi a^{2} e}{D}-2 \pi a(2 N H+1)-4 \pi e a(2 N V+1)\right]\end{array}$} \\
\hline$\frac{\partial \dot{G}}{\partial \dot{e}}$ & \multicolumn{2}{|c|}{$\begin{array}{l}\text { For prolate spheroid } \\
\frac{\partial \dot{G}}{\partial \dot{e}}=\gamma_{s}\left[\frac{2 \pi a^{2}}{\sqrt{e^{2}-1}}\left(2 e-\frac{e^{3}}{e^{2}-1}\right) \sin ^{-1}\left(\frac{\sqrt{e^{2}-1}}{e}\right)+\frac{2 \pi a^{2} e}{e^{2}-1}\right]+\gamma_{g b}\left[\frac{8 \pi a^{3} e}{3 D}-2 \pi a^{2}(2 N V+1)+4 \pi D^{2} \sum_{i=1}^{N V} i^{2}-\frac{4 \pi D^{2}}{e^{3}} \sum_{i=1}^{N H} i^{2}\right. \\
\text { For oblate spheroid } \\
\frac{\partial \dot{G}}{\partial \dot{e}}=\gamma_{s}\left[\frac{2 \pi a^{2}}{\sqrt{1-e^{2}}}\left(2 e+\frac{e^{3}}{1-e^{2}}\right) L n\left(\frac{1+\sqrt{1-e^{2}}}{e}\right)-\frac{2 \pi a^{2} e}{1-e^{2}}\right]+\gamma_{g b}\left[\frac{8 \pi a^{3} e}{3 D}-2 \pi a^{2}(2 N V+1)+4 \pi D^{2} \sum_{i=1}^{N V} i^{2}-\frac{4 \pi D^{2}}{e^{3}} \sum_{i=1}^{N H} i^{2}\right]\end{array}$} \\
\hline$\frac{\partial \dot{G}}{\partial \dot{D}}$ & \multicolumn{2}{|l|}{$\frac{\partial \dot{G}}{\partial \dot{D}}=\gamma_{g b}\left[\frac{4 \pi D}{e^{2}} \sum_{i=1}^{N H} i^{2}+8 \pi e D \sum_{i=1}^{N V} i^{2}-\frac{2 c^{2} h}{D^{2}}-\frac{c^{3}}{D^{2}}\right]$} \\
\hline $\begin{array}{l}\frac{\partial^{2} \Psi}{\partial \dot{a}^{2}} \\
=\frac{\partial^{2} \Psi_{G B}}{\partial \dot{a}^{2}}+\frac{\partial^{2} \Psi_{S}}{\partial \dot{a}^{2}}\end{array}$ & $\begin{array}{l}\frac{\partial^{2} \Psi_{G B}}{\partial \dot{a}^{2}}=\frac{2 \pi^{2} a^{4} e^{2} \xi}{c^{4} M_{G B} \Omega \delta_{G B}} \\
\frac{\partial^{2} \Psi_{S}}{\partial \dot{a}^{2}}=\left[\frac{1}{\pi M_{S} \Omega \delta_{S}} \int_{0}^{a e}\left(\frac{\partial j_{S i}^{V}}{\partial \dot{a}}\right)^{2} \frac{\sqrt{a^{2}+z^{2}\left(e^{-4}-e^{-2}\right)}}{\left(a^{2}-z^{2} e^{-2}\right)} d z\right]\end{array}$ & $\begin{array}{l}\text { where } \xi=F_{o} \eta_{o}^{2}+2 \sum_{i=1}^{N H} F_{i} \eta_{i}^{2} \\
\eta_{o}=\frac{F_{i}}{F_{i}+\sum_{i=1}^{N H} 2 F_{o}}\end{array}$ \\
\hline $\begin{array}{l}\frac{\partial^{2} \Psi}{\partial \dot{a} \partial \dot{e}} \\
=\frac{\partial^{2} \Psi_{G B}}{\partial \dot{a} \partial \dot{e}}+\frac{\partial^{2} \Psi_{S}}{\partial \dot{a} \partial \dot{e}}\end{array}$ & $\begin{array}{l}\frac{\partial^{2} \Psi_{G B}}{\partial \dot{e} \partial \dot{a}}=\frac{\partial^{2} \Psi_{G B}}{\partial \dot{a} \partial \dot{e}}=\frac{2 \pi^{2} a^{5} e \xi}{3 c^{4} M_{G B} \Omega \delta_{G B}} \\
\frac{\partial^{2} \Psi_{\mathrm{s}}^{\mathrm{o}}}{\partial \dot{\mathrm{e}} \dot{\mathrm{a}}}=\frac{\partial^{2} \Psi_{\mathrm{s}}^{\mathrm{o}}}{\partial \dot{\mathrm{a}} \dot{\mathrm{e}}}=\frac{1}{\pi \mathrm{M}_{\mathrm{S}} \Omega \delta_{\mathrm{s}}} \int_{0}^{\mathrm{ae}}\left(\frac{\partial j_{S}^{V}}{\partial \dot{a}}\right)\left(\frac{\partial j_{S}^{V}}{\partial \dot{e}}\right) \frac{\sqrt{\mathrm{a}^{2}+\mathrm{z}^{2}\left(\mathrm{e}^{-4}-\mathrm{e}^{-2}\right)}}{\left(\mathrm{a}^{2}-\mathrm{z}^{2} \mathrm{e}^{-2}\right)} \mathrm{dz}\end{array}$ & $\begin{aligned} & \eta_{i}=\frac{F_{o}}{F_{i}+\sum_{i=1}^{N H} 2 F_{o}} \\
& \frac{\partial j s}{\partial \dot{a}}=2 \pi e a^{2}-2 \pi a z-\frac{2 \pi^{2} e a^{4}}{c^{2}}+\frac{2 \pi^{2} a^{2} z^{2}}{e c^{2}}\end{aligned}$ \\
\hline $\begin{array}{l}\frac{\partial^{2} \Psi}{\partial \dot{e}^{2}} \\
=\frac{\partial^{2} \Psi_{G B}}{\partial \dot{e}^{2}}+\frac{\partial^{2} \Psi_{S}}{\partial \dot{e}^{2}}\end{array}$ & $\begin{array}{l}\frac{\partial^{2} \Psi_{G B}}{\partial \dot{e}^{2}}=\frac{2 \pi^{2} a^{6} \xi}{9 c^{4} M_{G B} \Omega \delta_{G B}} \\
\frac{\partial^{2} \Psi_{\mathrm{S}}^{\mathrm{o}}}{\partial \dot{\mathrm{e}}^{2}}=\frac{1}{\pi \mathrm{M}_{\mathrm{S}} \Omega \delta_{\mathrm{S}}} \int_{0}^{\mathrm{ae}}\left(\frac{\partial j_{S}^{V}}{\partial \dot{a}}\right)^{2} \frac{\sqrt{\mathrm{a}^{2}+\mathrm{z}^{2}\left(\mathrm{e}^{-4}-\mathrm{e}^{-2}\right)}}{\left(\mathrm{a}^{2}-\mathrm{z}^{2} \mathrm{e}^{-2}\right)} \mathrm{dz}\end{array}$ & $\frac{\partial j_{s}^{V}}{\partial \dot{e}}=\frac{2 \pi a^{3}}{3}-\frac{2 \pi z^{3}}{3 e^{3}}-\frac{2 \pi^{2} a^{5}}{3 c^{2}}+\frac{2 \pi^{2} a^{3} z^{2}}{3 e^{2} c^{2}}$ \\
\hline$\frac{\partial^{2} \Psi}{\partial \dot{D}^{2}}$ & \multicolumn{2}{|l|}{$\frac{\partial^{2} \Psi_{m i g}}{\partial \dot{D}^{2}}=\frac{A_{G B}}{4 m_{m}}$} \\
\hline
\end{tabular}


Table 3 Material property input data.

\begin{tabular}{|l|l|l|l|}
\hline Property & Units & Value & Sources \\
\hline$D_{s o}$ & $\mathbf{m}^{2} \mathbf{s}^{-1}$ & $1.0 \times 10^{-3}$ & {$[18,28]$} \\
\hline$Q_{s}{ }^{a}$ & $\mathbf{J m o l}^{-1}$ & $3.14 \times 10^{5} \sim 5.31 \times 10^{5}$ & {$[28,29]$} \\
\hline$D_{g b o}$ & $\mathbf{m}^{2} \mathbf{s}^{-2}$ & $1.0 \times 10^{-3}$ & {$[24]$} \\
\hline$Q_{g b}$ & $\mathbf{J m o l}^{-1}$ & $3.7 \times 10^{5}$ & {$[24]$} \\
\hline$\gamma_{s}$ & $\mathbf{J m}^{-2}$ & 0.3 & {$[18]$} \\
\hline$\gamma_{g b}$ & $\mathbf{J m}^{-2}$ & 0.15 & {$[18]$} \\
\hline$\Omega$ & $\mathbf{m}^{3}$ & $3.374 \times 10^{-29}$ & {$[18,28]$} \\
\hline$\delta_{s}$ & $\mathbf{m}$ & $3.231 \times 10^{-10}$ & {$[18,28]$} \\
\hline$\delta_{g b}$ & $\mathbf{m}$ & $6.462 \times 10^{-10}$ & {$[18,28]$} \\
\hline$\xi_{\text {imp }}{ }^{b}$ & & $1 \times 10^{3}$ & {$[18,28]$} \\
\hline$\xi_{m}{ }^{c}$ & & $5 \times 10^{4}$ & {$[18,28]$} \\
\hline
\end{tabular}

a. $Q_{s}$ is $314 \mathrm{~kJ} / \mathrm{mol}$ according to [28], $531 \mathrm{~kJ} / \mathrm{mol}$ in [29]

b. A correction factor to account for the increase of sintering rate due to impurity segregation at grain boundary.

c. A correction factor to account for the drag force exerted by segregated solutes at grain boundaries. 
Table 4 Grain growth kinetic parameters for 3 YSZ and 8YSZ films.

\begin{tabular}{|c|c|c|c|c|}
\hline Film & $m$ & $k_{o}$ & $G_{o}$ & $Q_{G}$ \\
\hline $3 Y S Z$ & 4 & $1.81 \times 10^{4}$ & $0.17 \mu \mathrm{m}$ & $327 \mathrm{~kJ} / \mathrm{mol}$ \\
\hline $8 Y S Z$ & 2 & $2.86 \times 10^{6}$ & $0.15 \mu \mathrm{m}$ & $331 \mathrm{~kJ} / \mathrm{mol}$ \\
\hline
\end{tabular}


Figure Captions

Fig.1 SEM images for films sintered at $1350^{\circ} \mathrm{C}$ for $1 \mathrm{~h}$ a) $3 \mathrm{YSZ}$ top surface, b) $8 \mathrm{YSZ}$ top surface, c) 8YSZ cross section.

Fig. 2 Large defect present on the top surface of $3 \mathrm{YSZ}$ film sintered at $1300^{\circ} \mathrm{C}$ for $1 \mathrm{~h}$.

Fig.3 Density achieved for 3YSZ and 8YSZ films under different sintering conditions.

Fig.4 Pore size changes for 3YSZ and 8YSZ films as a function of density.

Fig.5 Evolution of pore elongation in 3YSZ and 8YSZ films (the hollow symbols are for 3 YSZ, solid symbols for 8YSZ; circles for those observed on top surfaces (TS) and stars for those observed on cross sections (CS))

Fig. 6 Evolution of degree of preferred orientation, $k$, of pores in 3YSZ and 8YSZ. When $k>0$ the pores are preferentially oriented with their long axis perpendicular to the film plane.

Fig.7 Grain size as a function of density for 3 YSZ and 8 YSZ films sintered at $1150-1350^{\circ} \mathrm{C}$ for up to $48 \mathrm{~h}$

Fig.8 A half unit cell for the sintering model showing a pore intersected by horizontal and vertical grain boundaries.

Fig.9 Pore shape evolution predicted by the sintering model, a) initial shape a sphere, b) initial shape an oblate spheroid.

Fig.10 Evolution of pore shape predicted by the sintering model and its sensitivity to relative rates of grain boundary to surface diffusion.

Fig.11 Pore size evolution predicted by the sintering model.

Fig. 12 Large defects present at the top surface of a 3 YSZ film sintered at a) $1200{ }^{\circ} \mathrm{C}$ for $10 \mathrm{~min}, \mathrm{~b})$ for an additional $10 \mathrm{~min}$ at $1300^{\circ} \mathrm{C}$.

Fig13 a) pore size distribution observed on cross sections of 3 YSZ films sintered at $1350{ }^{\circ} \mathrm{C}$ for $5 \mathrm{~min}$; b) the modelling predictions of pore elongation for $3 \mathrm{YSZ}$ for different relative rates of grain boundary to surface diffusion: $R_{D F}=0.016$ (corresponds to $Q s=314 \mathrm{~kJ} / \mathrm{mol}$ ), $\mathrm{R}_{\mathrm{DF}}=0.22(\mathrm{Qs}=350 \mathrm{~kJ} / \mathrm{mol}), \mathrm{R}_{\mathrm{DF}}=1(\mathrm{Qs}=370 \mathrm{~kJ} / \mathrm{mol}), \mathrm{R}_{\mathrm{DF}}=1653(\mathrm{Qs}=470 \mathrm{~kJ} / \mathrm{mol})$; the star symbols are experimental results (also shown in Fig.5). 


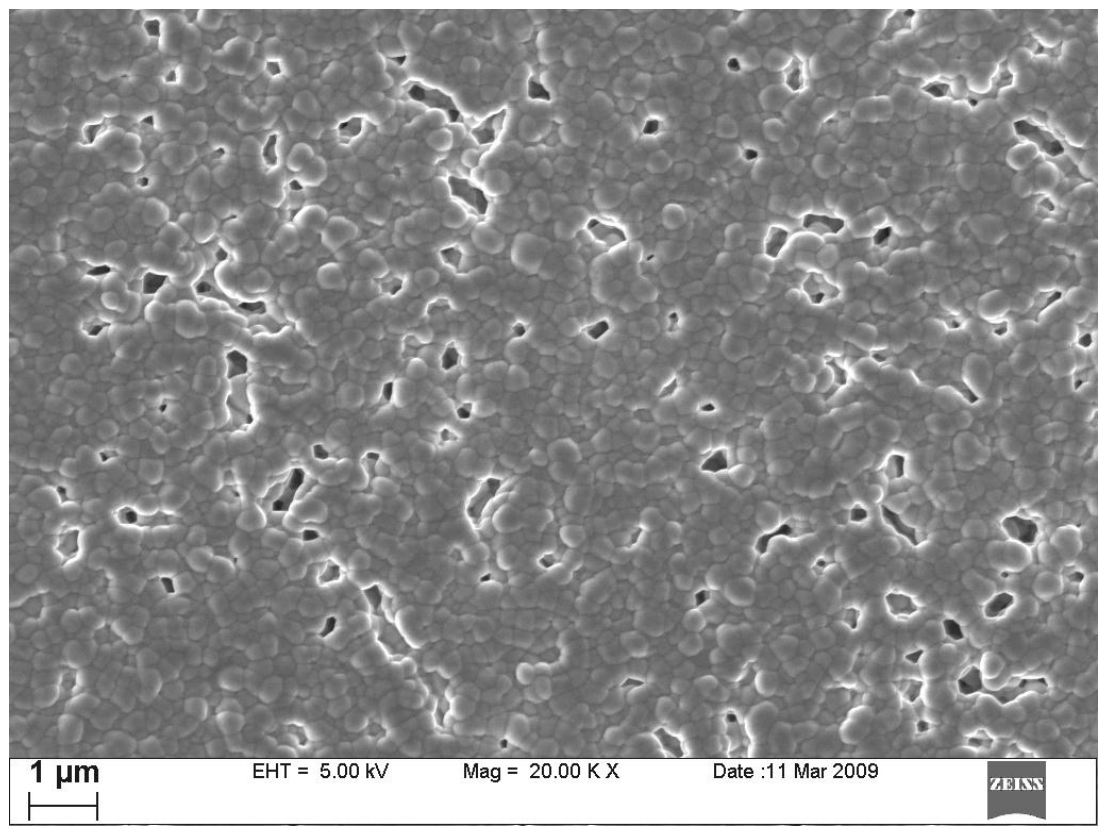

Fig.1a

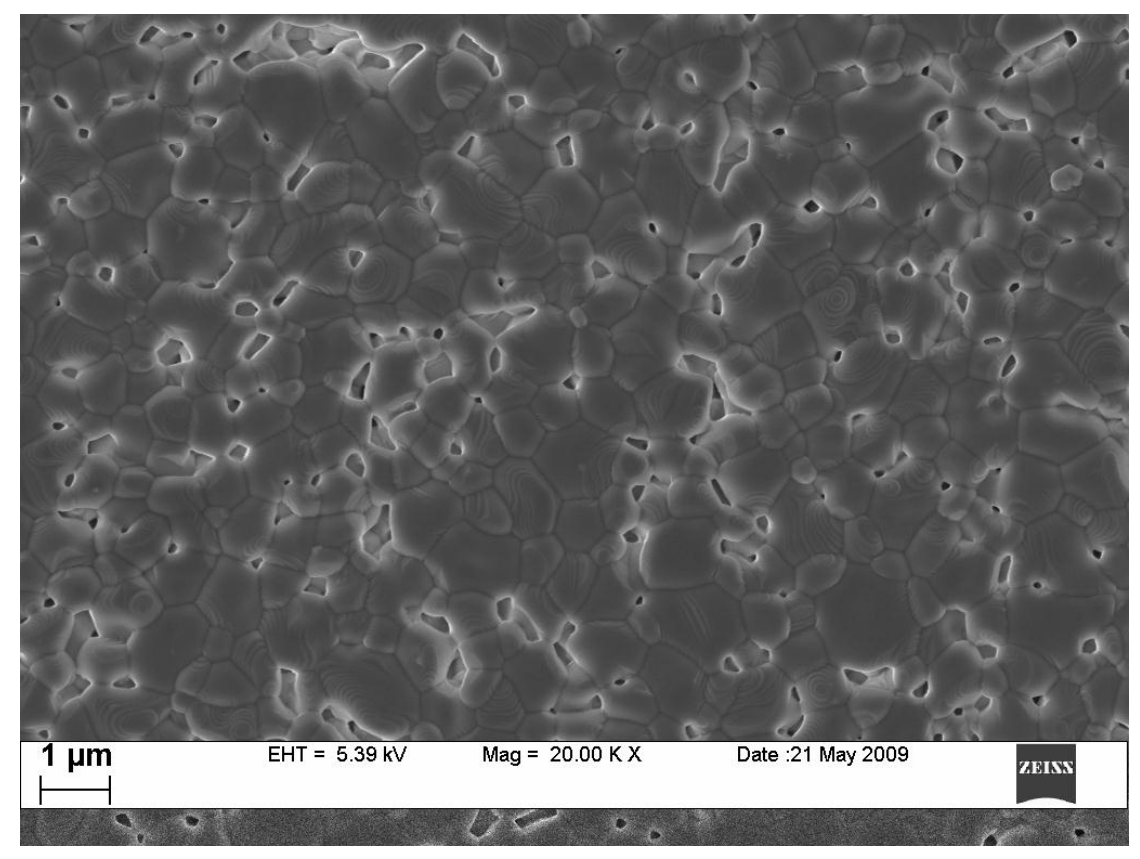


Fig. 1b

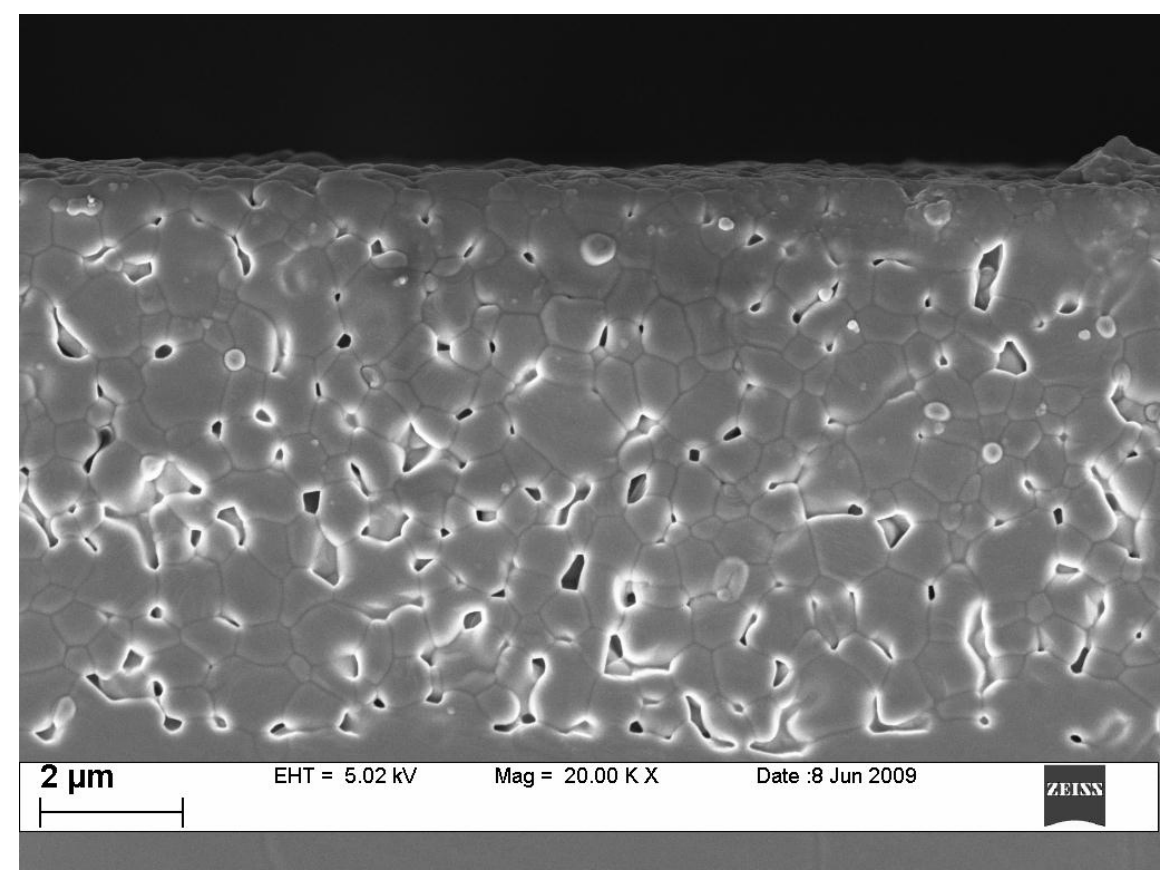

Fig.1c 


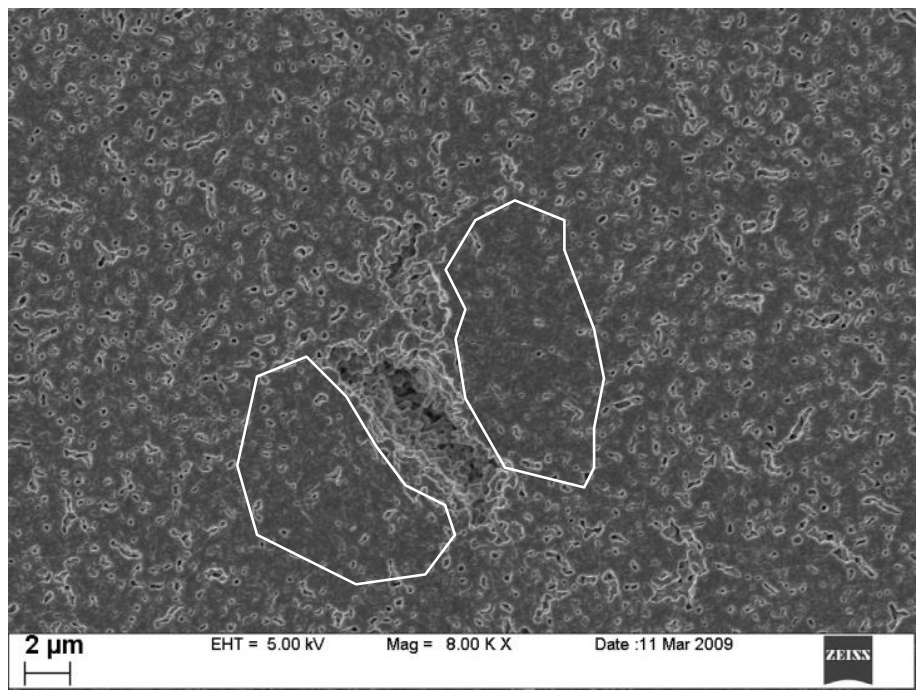

Fig 2

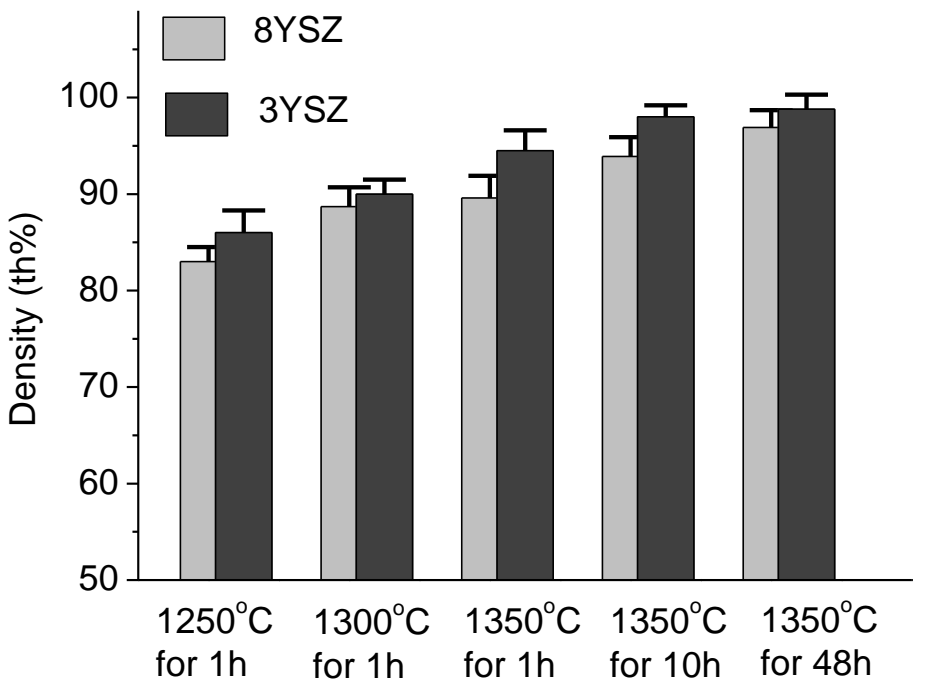


Fig. 3

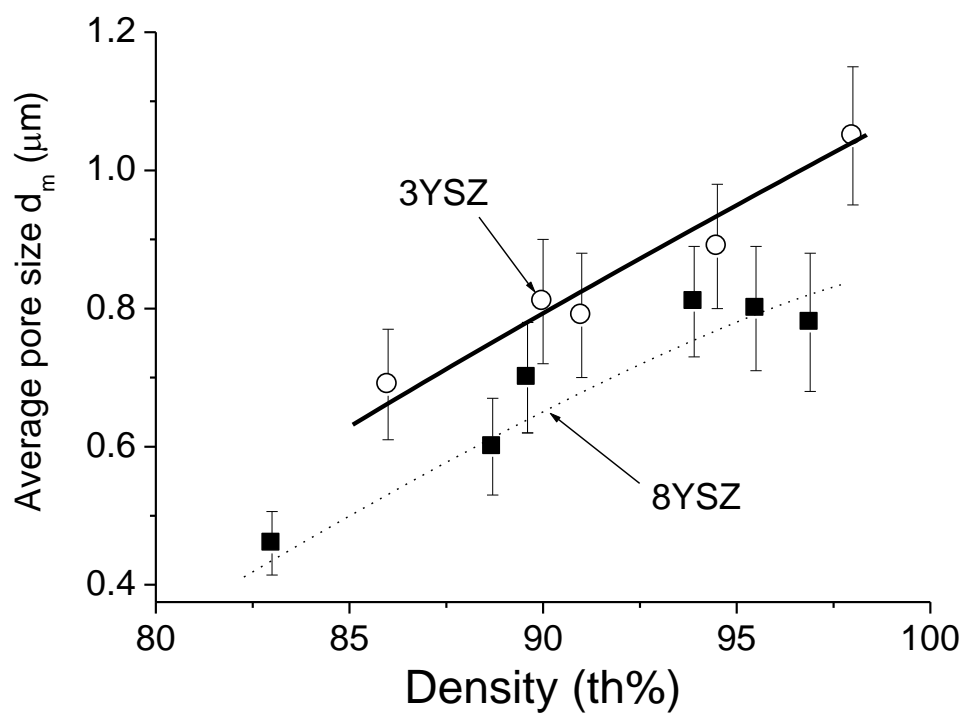

Fig.4

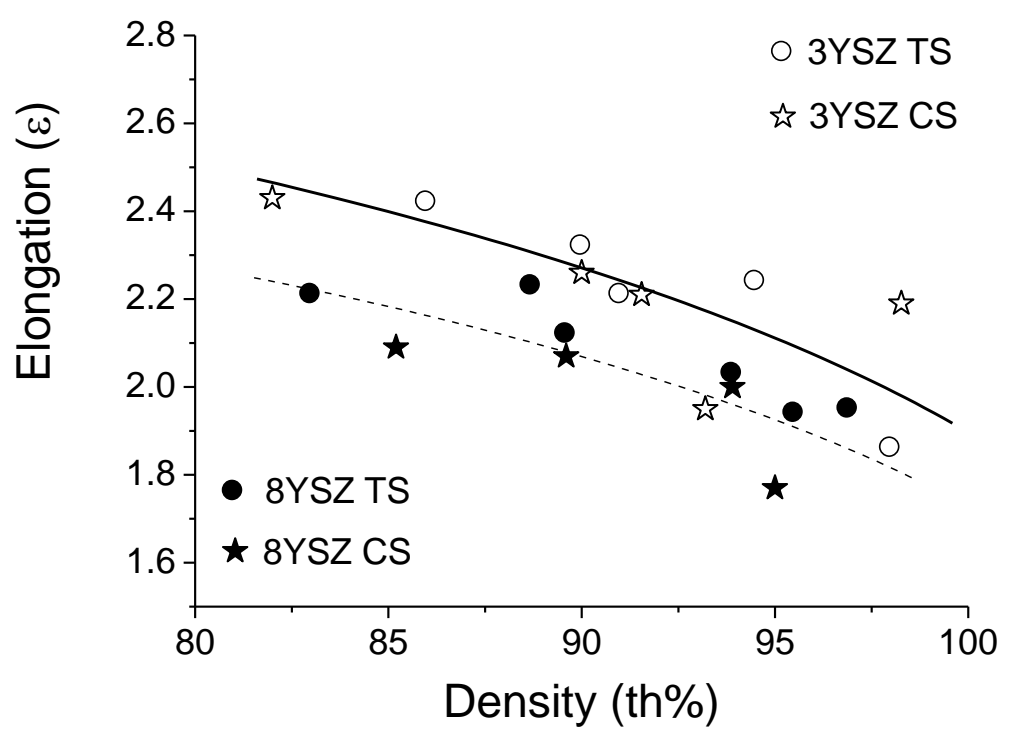

Fig. 5 


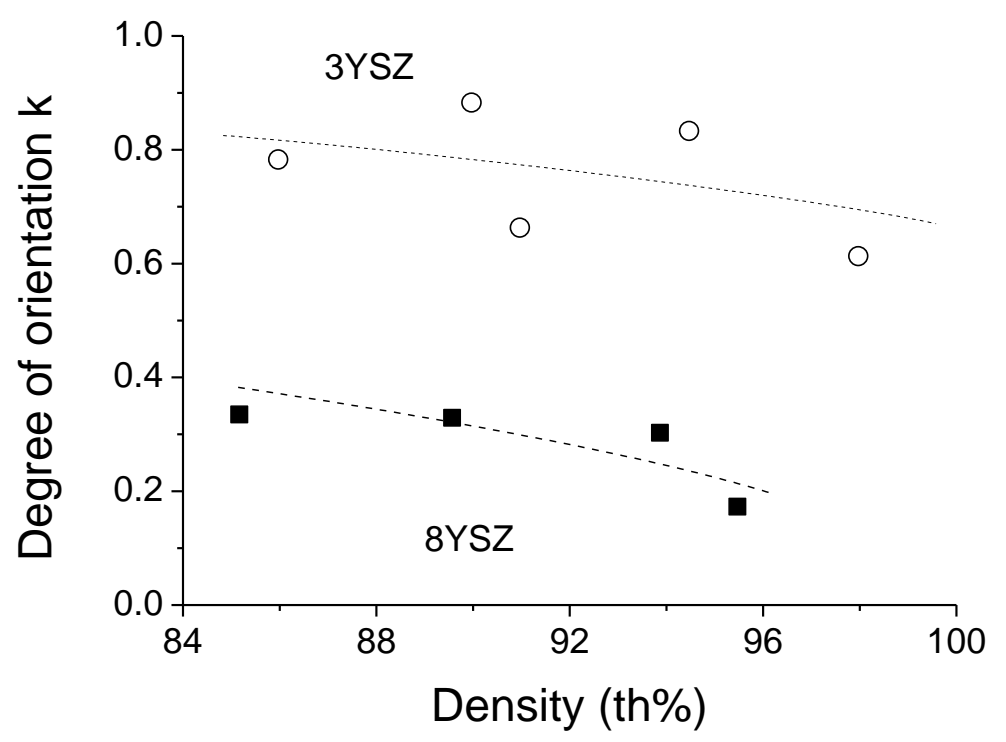

Fig. 6

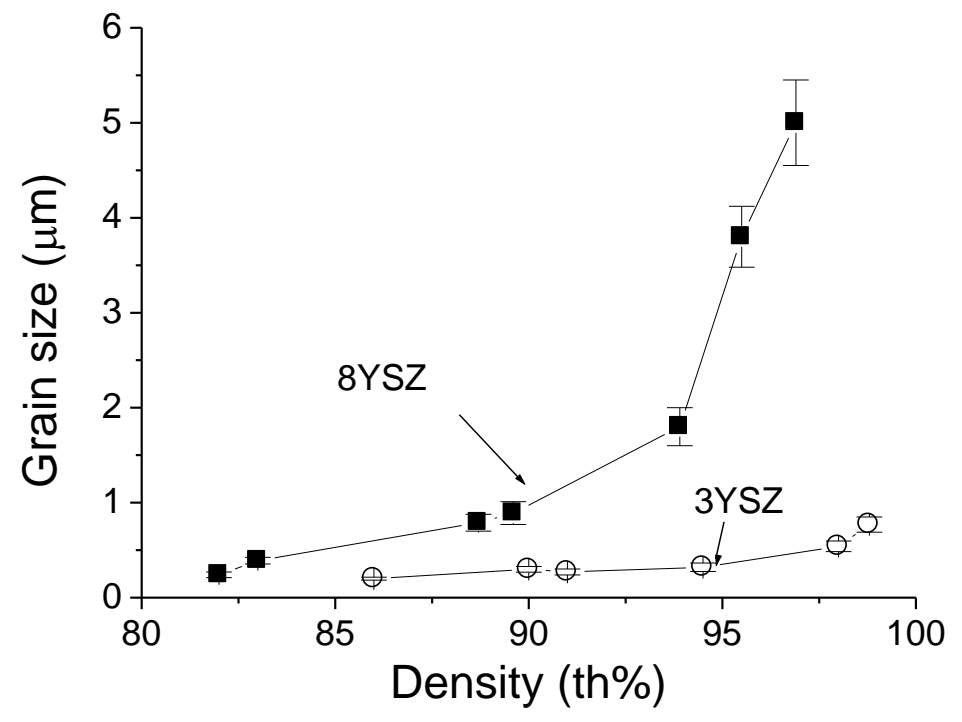

Fig.7 


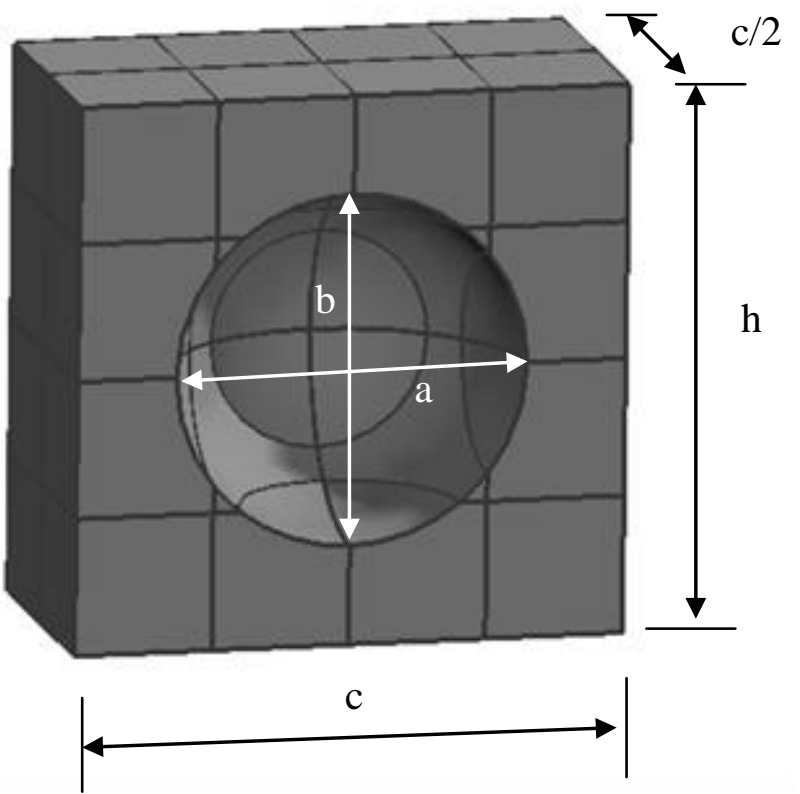

Fig. 8 


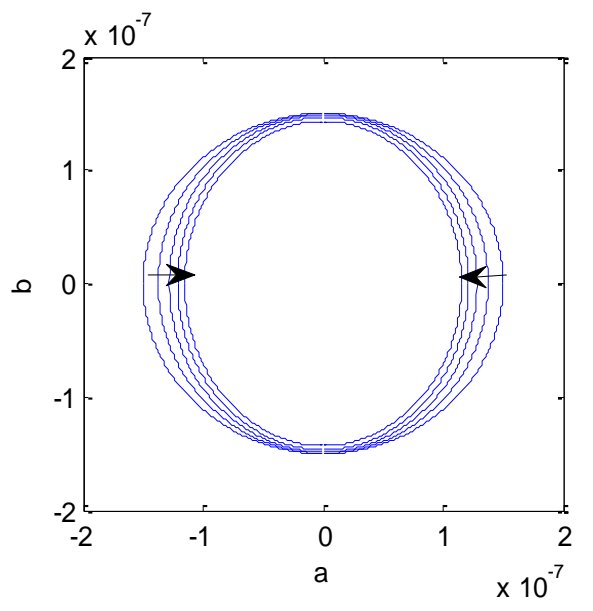

a

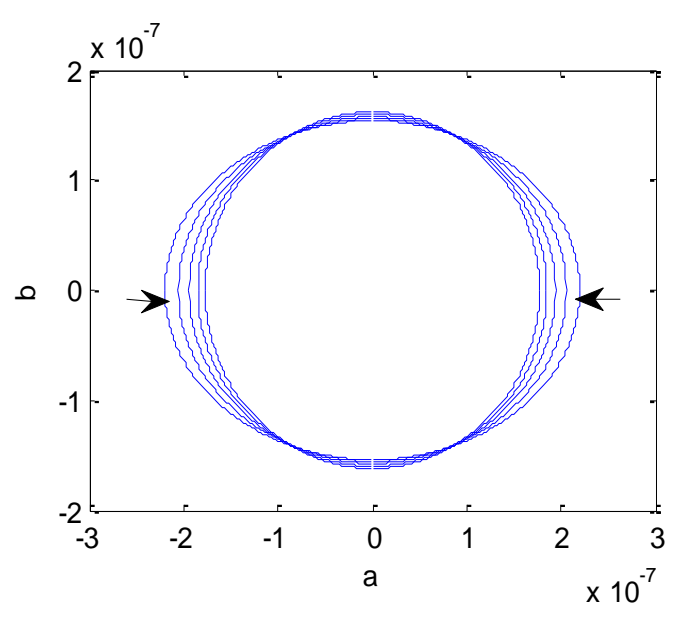

b

Fig.9 


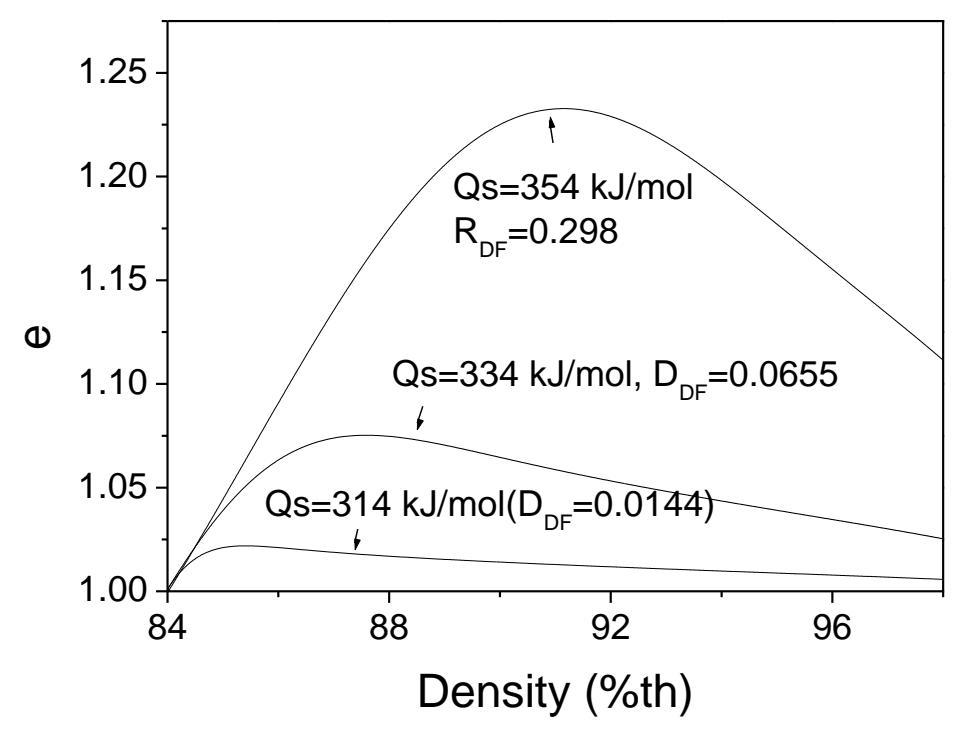

Fig.10

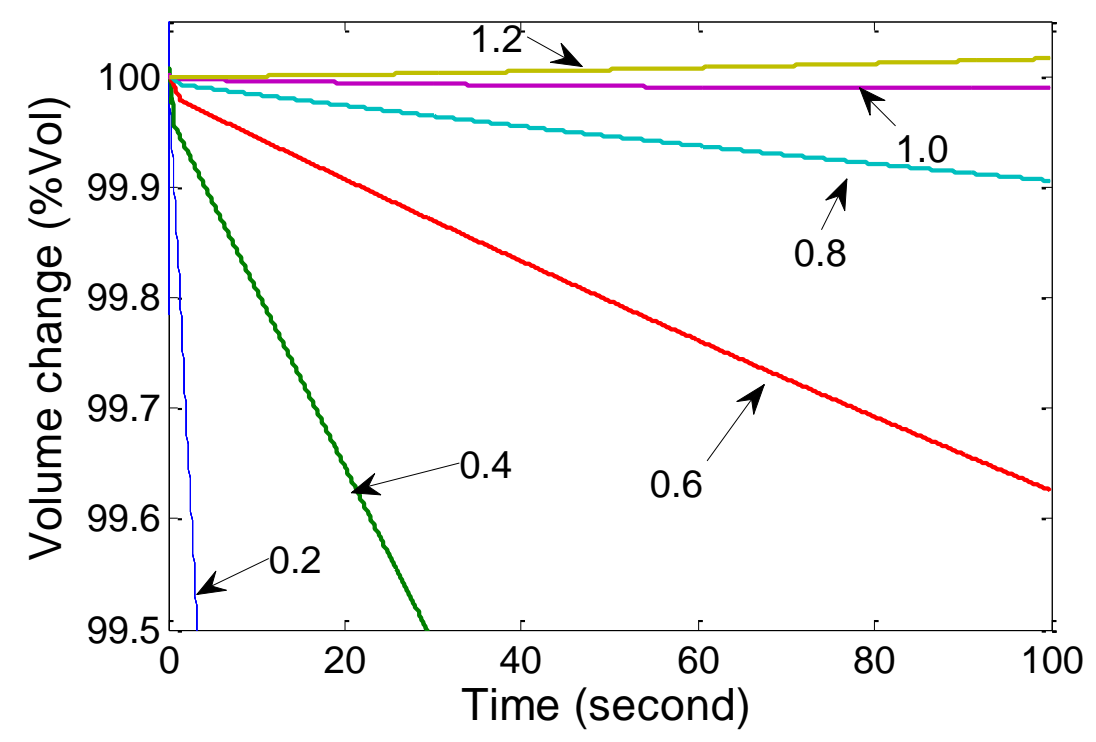

Fig.11 


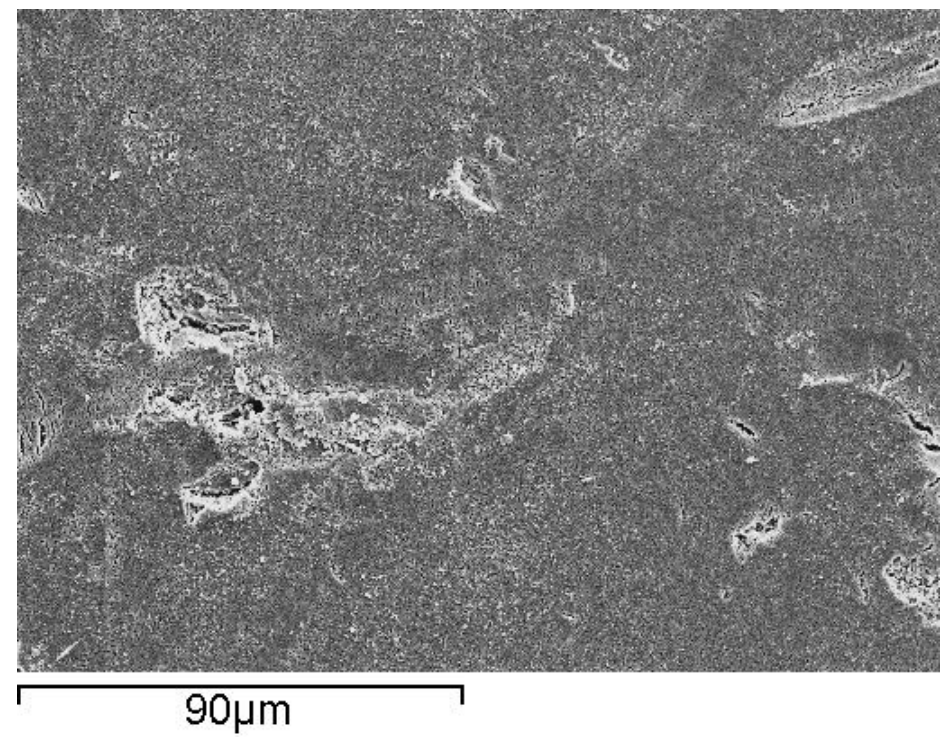

.a

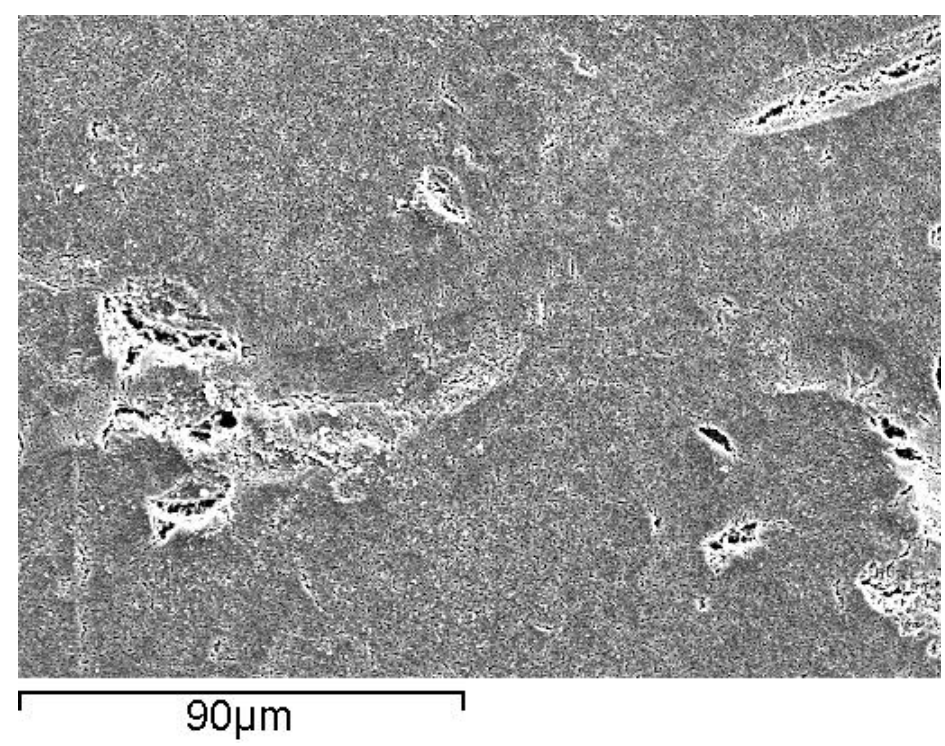

b

Fig.12 


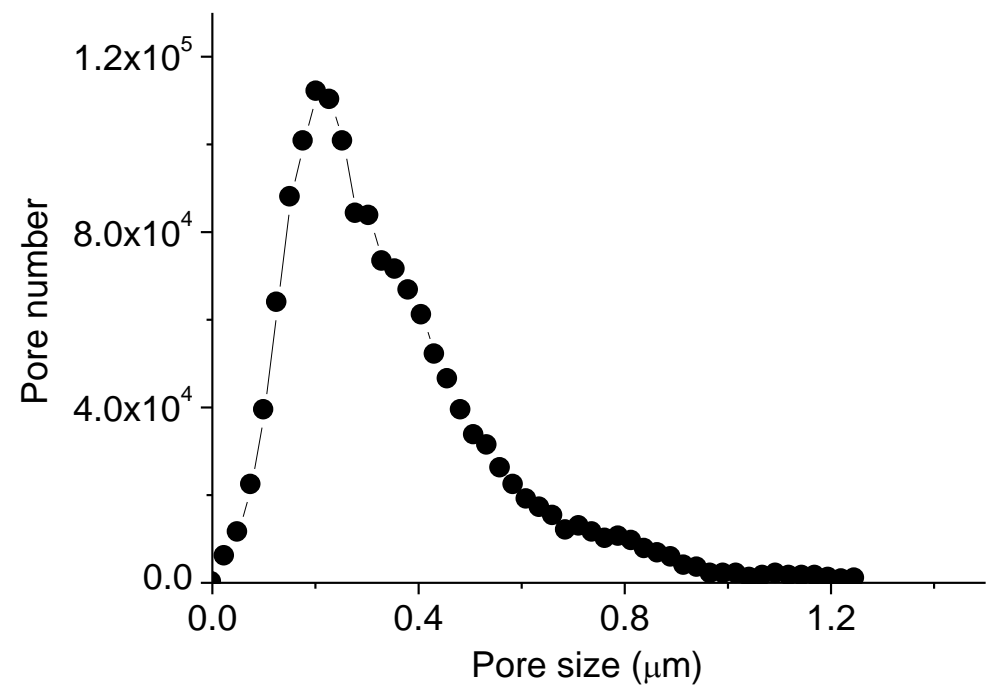

Fig.13a

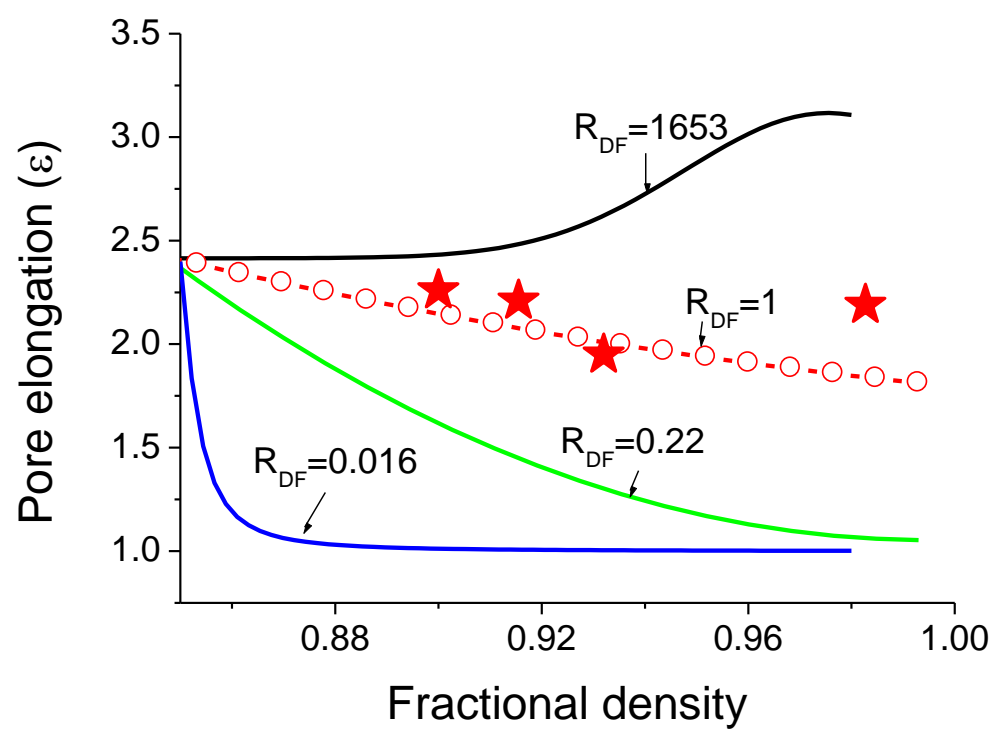

Fig. 13b 